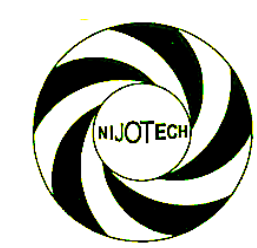

Nigerian Journal of Technology (NIJOTECH)

Vol. 39, No. 3, July 2020, pp. 860 - $\mathbf{8 7 0}$

Copyright@ Faculty of Engineering, University of Nigeria, Nsukka,

Print ISSN: 0331-8443, Electronic ISSN: 2467-8821 www.nijotech.com

http://dx.doi.org/10.4314/njt.v39i3.28

\title{
MEDIUM TERM ELECTRICAL LOAD FORECAST OF ABUJA MUNICIPAL AREA COUNCIL USING ARTIFICIAL NEURAL NETWORK METHOD
}

\author{
E. C. Ashigwuike ${ }^{1}$, A. R. A. Aluya ${ }^{2}{ }^{2}$, J. E. C. Emechebe ${ }^{3}$ and S. A. Benson ${ }^{4}$ \\ 1,4, Department of Electrical Electronic Engineering, University of ABuja, ABuja FCT, NIGERIA \\ 2, TRANSMISSION COMPANY OF NIGERIA (TCN), ABUJA F.C.T, NIGERIA \\ 3. Federal Radio Corporation OF Nigeria, ABUjA F.C.T, NIGERIA \\ Email addresses: ${ }^{1}$ evans.ashigwuike@uniabuja.edu.ng, ${ }^{2}$ aluyaresepectandrew@gmail.com, \\ 3 jonasemechebe@gmail.com, 4 iamsteveben@gmail.com
}

\begin{abstract}
This paper presents a medium-term electric load forecast for Abuja Municipal Area Council (AMAC) distribution network based on Artificial Neural Network (ANN). The technique results are compared with that of a conventional method (Multiple Linear Regression method), for the same data. The $A N N$ proposed method takes into account the effect of temperature, time, population growth rate and the activities of different regions of city areas regarding lifestyle and types of consumers. The data of monthly to annual peak values are collected for the period from 2012 to first quarter of 2018. Hence, the Artificial Neural Network method presented a result with average MAPE of 0.00197 while the multiple linear regression having an average MAPE of 0.004545 . The $R$-Value deviation was $8.06 \%$ and $34.42 \%$ for ANN and MLR methods respectively.
\end{abstract}

Keywords: Artificial Neural Network, Forecast, Load, Energy Demand, Capacity Allocation, Percentage error, Forecasting Accuracy.

\section{INTRODUCTION}

Electric load forecasting has in recent times received great and increasing attention because it is seen as a very vital component of power generation and management systems, in cities and countries with fast growing rate of infrastructural development [1]. Accurate load forecasting will aid both the electricity generation companies and the distribution companies to make unit commitment decisions with regards to power, load switching, voltage control, network reconfiguration, and infrastructure development [2]. It also reduces the generation cost and increases reliability of power systems. Because of how important load demand forecasting has become overtime, research has been intensified in this field, with different models being developed and employed by different researchers for short-term, medium-term and long-term load forecasting over the last decade. Some of these developed models are the regressionbased models, time series approaches, Box-Jenkins model, Kalman filters, expert system techniques, artificial neural network (ANN) models, fuzzy logic, wavelet transforms and fuzzy neural network (fNN) structures. Some other models combine two or more of these models listed above with the sole aim of increasing the accuracy of the model [3 - 7].

There is a high influx of people from the rural regions and other parts of Nigeria to Abuja, and this has consequently led to an excessive demand of electricity as a result of the fast growing rate of industries, economic and infrastructural development, and an increase in the population of the residence thereby causing increased load demand without commensurate increase in power supply hence power epilepsy, floatation and power outages. The major task of electricity companies is to accurately forecast based on the previous data generated manually, the energy requirements of the people at all times, and based on such forecast, utility companies coordinate their resources to meet the forecasted load demand, thereby engaging a least cost energy management plan subject to numerous uncertainties and follow it up. Therefore, this work 
provides an effective way of forecasting electric load targeted at enhances reliable power to the populace.

\section{LITERATURE REVIEW}

Load forecasting is indispensable in the operation and scheduling of a utility company. Load forecasting assists an electric utility in making vital decisions including purchasing and generating electric power, load switching, and infrastructure development [8]. Since its inception, so many techniques have been employed over time for predicting the load demand of a given geographical area. According to Singh et al. [9], load forecasting techniques can be classified based on the duration of forecasting into Short term, Medium term and Long term load forecasting. Short term load demand forecasting is the prediction done for like one hour or more but not up to 24 hours, medium term load demand forecasting is the type of forecasting lasting from 2 days till around 2 weeks or a month as the case maybe, while the long term load forecasting lasts up to 6 months to a year or more. Arjun Baliyan, et al [10] reviewed lately available research work on different alternates of artificial neural network in the aspects of short term load forecasting. Particularly, the hybrid networks which is a mishmash of stochastic learning techniques using neural such as particle swarm optimization (PSO), genetic algorithm (GA), etc. which has stayed effectively applied for short term load forecasting. Amera Ismail et al further in [11] analyze and deliberate an inclusive methodology for Short Term Load Forecasting with artificial neural network. The suggested architectures were trained and tested with prior two years definite load data acquired. In the study, four ANN models are applied and validated with practical precision on real electric load generation output data. The first and second model are to forecast values of one forward day and seven days, though the third and fourth models are also to forecast values of subsequent and seven days, regarding the extent of period of disconnected time. A forecasting performance measure such as the absolute mean error AME has been presented for each model. D.C. Park et al [12] presented a neural network methodology to electric load predicting. In order to deliver the forecasted load, ANN interpolate among the load and temperature data in a training data set using the average absolute errors of the onehour and 24-hour forward forecasts in the test on actual utility data achieved at $1.40 \%$ and $2.06 \%$, respectively. This compares with an average error of
$4.22 \%$ for 24 hour forecasts with a currently used forecasting technique applied to the same data.

The traditional techniques were used in the early days of load demand forecasting with preference to short term interval. Modification of these traditional techniques and development of more advanced tools have made prediction in different fields of study more effective and accurate and extend to the forecast of medium time interval. The traditional forecasting techniques include regression, multiple regression, iterative re-weighted least-squares, and exponential smoothing techniques $[1,7,9]$.

\section{MATERIAL AND METHODS}

This work is focused on the prediction of Monthly Load distribution of Abuja Municipal Area Council (AMAC) using both the Artificial Neural Network technique and the multiple regression technique. The results of the two methods employed were compared to ascertain which is more efficient and accurate based on the error calculations from the two techniques.

The artificial neural network has different architectures but for this work the feed forward NARX Neural network was used for the load demand prediction due to it flow of information in one direction from the input layer to the output layer. This network was trained to learn the relationship between the Network inputs and the desired output at a reduced error rate so as to correctly predict the future load demand when the inputs are fed. It is most times used because it is relatively simple and powerful, making use of gradient descent to reduce the mean-square error between the desired output and the actual output for every given input into the network. While in the unsupervised learning, there is no learning algorithm for the network, there is no feedback on the difference between the desired and resulting outputs, with the system adjusting itself into a useful configuration [13]. In this work, the feed forward NARX NN was used with back propagation training technique to predict the monthly load of Abuja Municipal Area council (AMAC). The general flowchart for the back propagation training algorithm is as shown in Figure 1. When applying artificial neural network technique to load forecasting, some important features are considered which improves the accuracy and the preciseness of the results obtained; one of these features is the network structure used for load forecasting which depend on the number of layers used.

Vol. 39, No. 3, July 2020 


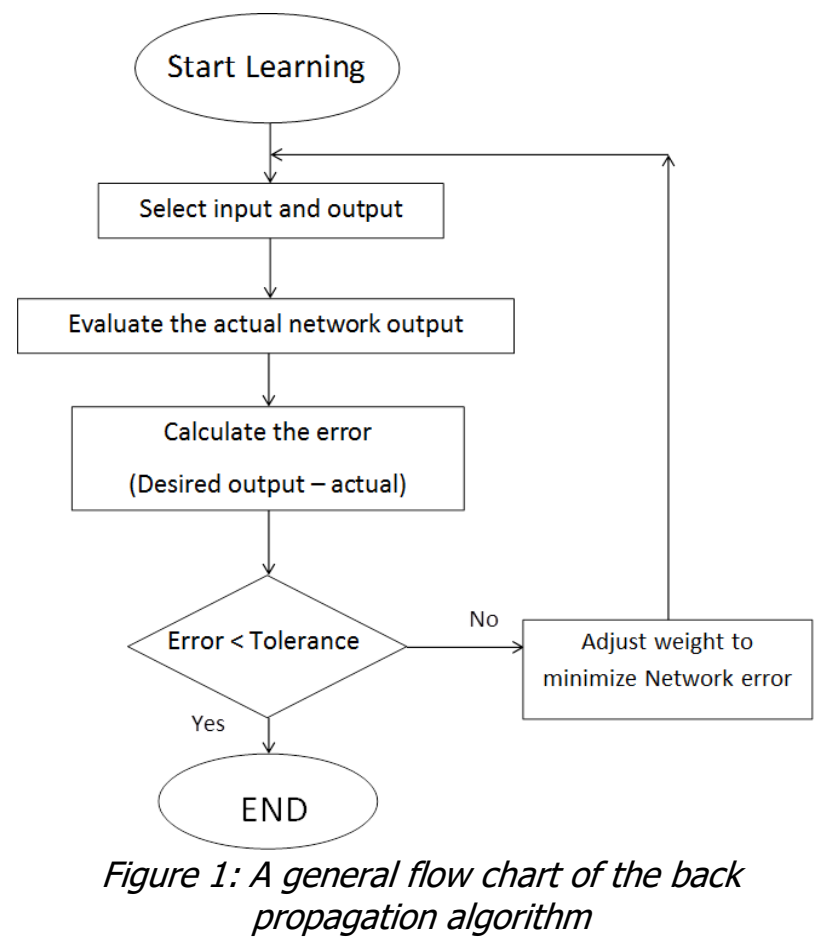

The most commonly used is the three layer network which is divided into input, hidden and output layers respectively. Another critical decision in the structure of the network is the number of hidden layers to be used. If there are many hidden layers in the network, the data used as input will only be memorized without the system forming a pattern for forecasting. The number of hidden layers to be used should be dependent on how difficult the task at hand becomes. The number of input and output neurons is determined by the number of input data, and then the number of output the designer wants. Since in this work we are interested in the monthly forecast of the electricity load for AMAC Abuja, the system was designed such that output will have an output for each of the twelve months that makes up a year [14].

\subsection{Data Collection and preparation}

The electrical load data of the area under study (Abuja Municipal Area Council (AMAC)) from 2012 till 2018 was obtained from the Abuja Electricity distribution company (AEDC), the population data for AMAC was obtained from world population review site, the growth rate was used to calculate the expected average population for year 2018-2020 with the growth for Abuja estimated at $6.1 \%$ per annum [15] and the temperature data from 2012 up to 2018 was also collected online from the Nigerian Institute of Meteorology database. The data was collated and prepared on an excel worksheet and then loaded into the Matlab workspace where it was

converted to matrix form and used for training the Artificial neural network (ANN).

\subsection{Design of the Non-Linear auto-regressive Network with exogenous input (NARX)}

This type of network is an auto-regressive exogenous (ARX) input based network which is mainly used for modelling time-series. As discussed in the literature review, the NARX model is defined by [13, eq. (1)]

$$
\begin{gathered}
y(t)=f(y(t-1), y(t-2), \ldots, Y(t-n), u(t-1), u(t \\
\left.-2), \ldots, u\left(t-n_{u}\right)\right)
\end{gathered}
$$

Using the NARX model, the next value of the dependent output signal was regressed on the previous values of an independent (exogenous) input. In this work, the NARX model was implemented using the feed forward neural network topology which allows the input and the output to be multidimensional. The NARX model has diverse application in different fields but in this work it was used as a predictor to forecast the electricity load demand of Abuja municipal area council (AMAC). The Series-Parallel architecture also known as the open loop was used during the training of the network, after which the parallel architecture also known as the closed loop was used for testing. The equations for the open loop and closed loop architectures respectively are given below [16, eq. (2) and (3)];

$$
\begin{gathered}
\hat{\mathrm{y}}(t+1)=F\left(y(t), y(t-1), \ldots, y\left(t-n_{y}\right), x(t+\right. \\
\left.1), x(t), x(t-1), \ldots, x\left(t-n_{x}\right)\right) \\
\hat{\mathrm{y}}(t+1)=F\left(\hat{\mathrm{y}}(t), \hat{\mathrm{y}}(t-1), \ldots, \hat{\mathrm{y}}\left(t-n_{y}\right), x(t+\right. \\
\left.1), x(t), x(t-1), \ldots, x\left(t-n_{x}\right)\right)
\end{gathered}
$$

$F(t)$ is the mapping function for the neural network and in this work, it is the logsigmoid function, where $\hat{y}(t+1)$ is the output of model at time ' $t$ ' for the time $t+1$ (i.e the value of $y$ for the time $(t+1)$. Then $\hat{y}(t)$, $\hat{y}(t-1), \ldots \hat{y}\left(t-n_{y}\right)$ represents the past outputs of the model. The true past values of the time series is given by $y(t), y(t-1), \ldots, y\left(t-n_{y}\right)$, it can also be called the desired output values. The inputs of the NARX model are $\mathrm{x}(\mathrm{t}+1), \mathrm{x}(\mathrm{t}), \ldots, \mathrm{x}\left(\mathrm{t}-\mathrm{n}_{\mathrm{y}}\right)$ and then $\mathrm{n}_{\mathrm{x}}$ is the number of input delays, $n_{y}$ is the number of output delays. To design NARX model with Matlab, the user interface of the neural network was opened with the 'nnstart' command which brings out the GUI as seen in Figure 3.2 and then clicking the 'time series app' button, or by going to the application tab in Matlab and then clicking on the "Neural Net. Time Series" icon, then the same GUI in Figure 2 will pop up. 


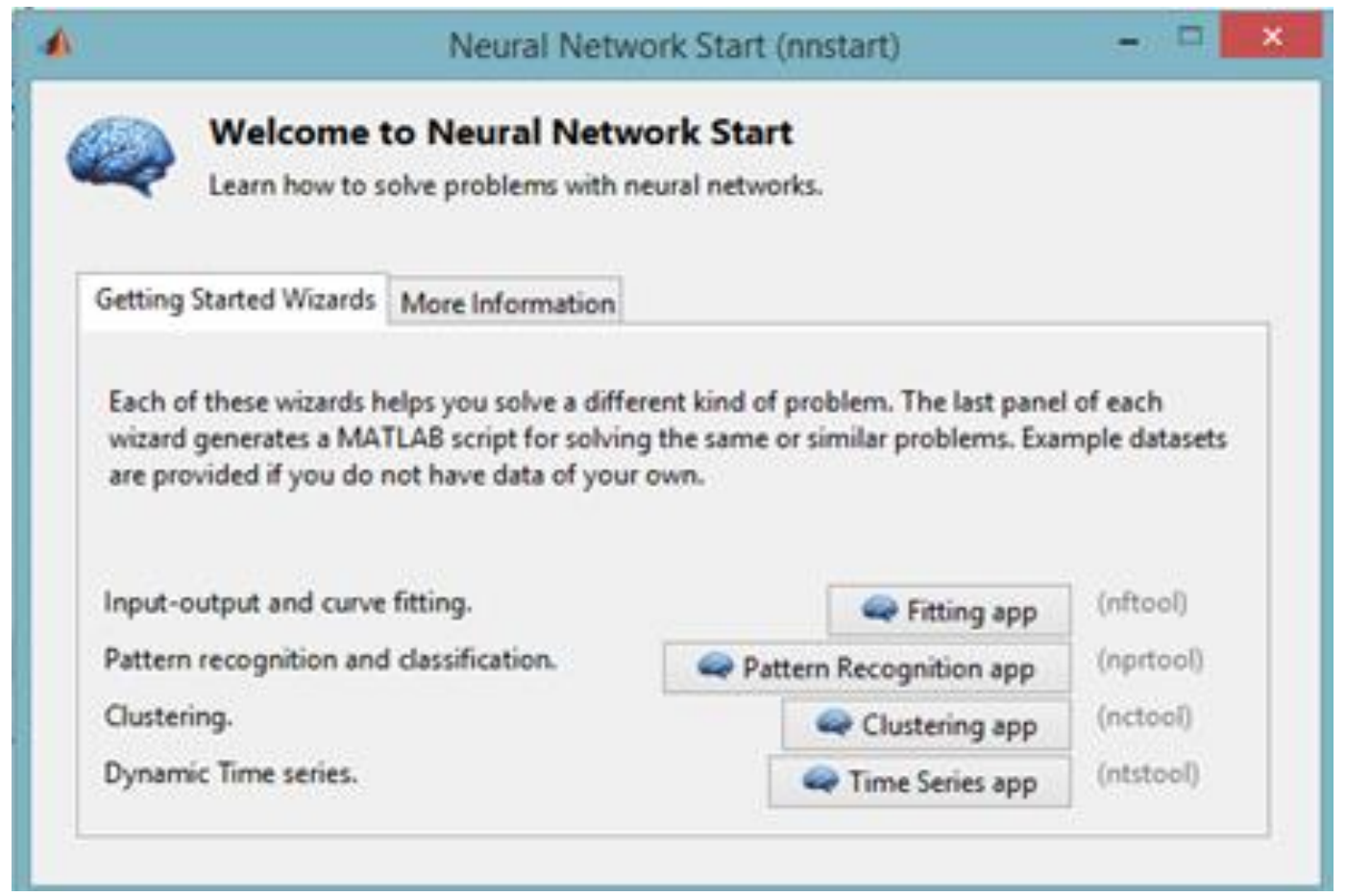

Figure 2: GUI showing different neural network tools

The NARX neural network model used for this work has three layers, with the Gaussian Log Sigmoid (logsigmoid) and pure Linear (purelin) functions used as activation (mapping) functions for the hidden and the output layers respectively. The mapping functions are known initially and are adjusted or modified during the training process. In NARX model, this modification or approximation is carried out by an internal structure called the Multi-layer perceptron (MLP) which allows the network to learn any form of continuous nonlinear mapping. The block diagram of the proposed NARX model is shown Figure 3.

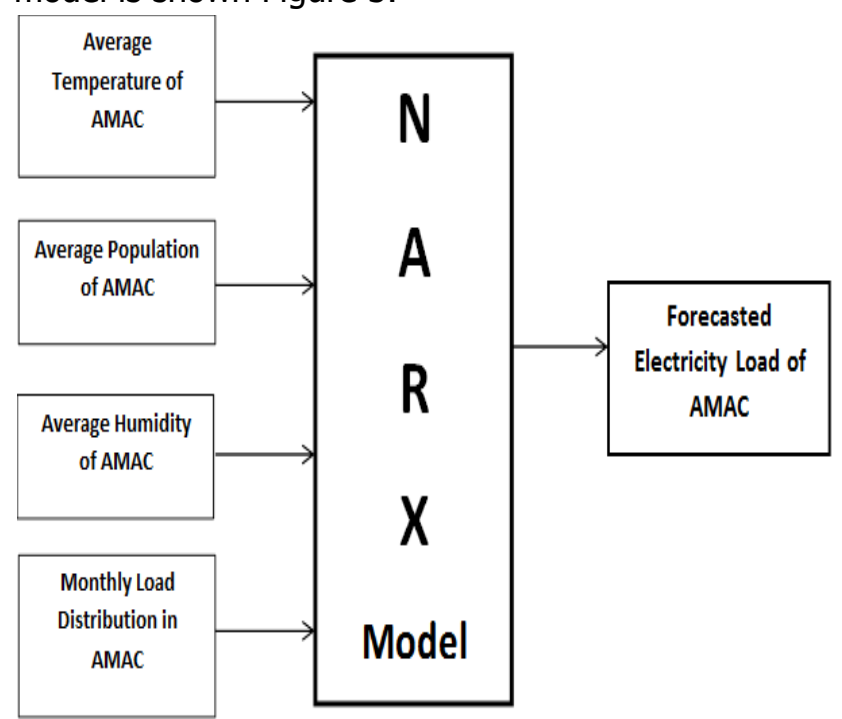

Figure 3: Block diagram of the proposed Load forecasting NARX model
From the block diagram of Figure 3, the previous average monthly temperature, the previous average monthly humidity and the population of Abuja Municipal Area council (AMAC) was fed into the NARX network as inputs, with the Previous Monthly Load distributions being fed as the target output.

\subsection{Choice of Input Variables}

Since there is no given standard stating the variables to be used when developing a model for load demand forecasting, the research chooses the input variables that have strong correlations to the load demand to develop his or her model. Therefore in this work, the dates in Months (Day indicator), average monthly temperature, average humidity, the population of the AMAC, and the load demand of AMAC from 2012 up to 2017 as input variables to train the neural network. This is because the network needs to know the kind of stimuli that affects the load needs of AMAC so as to make a better decision on the kind of input variable to select. A total of 72 monthly observations showing 72 different sample data of the Average temperature, Humidity, population of AMAC and the months were used as input to the network. The data was collated from 2012 to 2017. The input was presented as a 72 $x 4$ matrix. Moreover, 72 sample data of the past System load was used as the target, presented as a $72 \times 1$ matrix. The 72 sample data were used for 
training and validation and then the data for 2018 observations was used for testing the network.

\subsubsection{Training rule and rate}

The back propagation method which is one of the best learning rules for prediction in the Matlab ANN was chosen so as to produce effective results and accuracy, as the back propagation learning technique constantly adjusts the weights of the inputs throughout the training period until the mean squared error is reduced. The period at which the learning should go on was also defined so as to reduce overshooting in error correction. Figure 4 shows the training interface in the Matlab software.

The parameters of the 3 layered feed forward neural network training used in this work are as given below:
1. Number of layers: 3 (Input, hidden and output)

2. Number of neurons in input layer : 20

3. Number of neurons in the output layer: 1

4. Data division: random (dividerand)

5. Activation function for the hidden layer: logsigmoid

6. Activation function for output layer: purelin

7. Training algorithm: Lavenberg-Marquardt(train $/ m)$

8. Number of input variables: 72

9. Number of delays: 2

10. Number of hidden neuron: this was adjusted until the optimum training performance was obtained.

The designed NARX-ANN model is as shown in Figure 5 , as obtained from Matlab at the time of the training/simulation

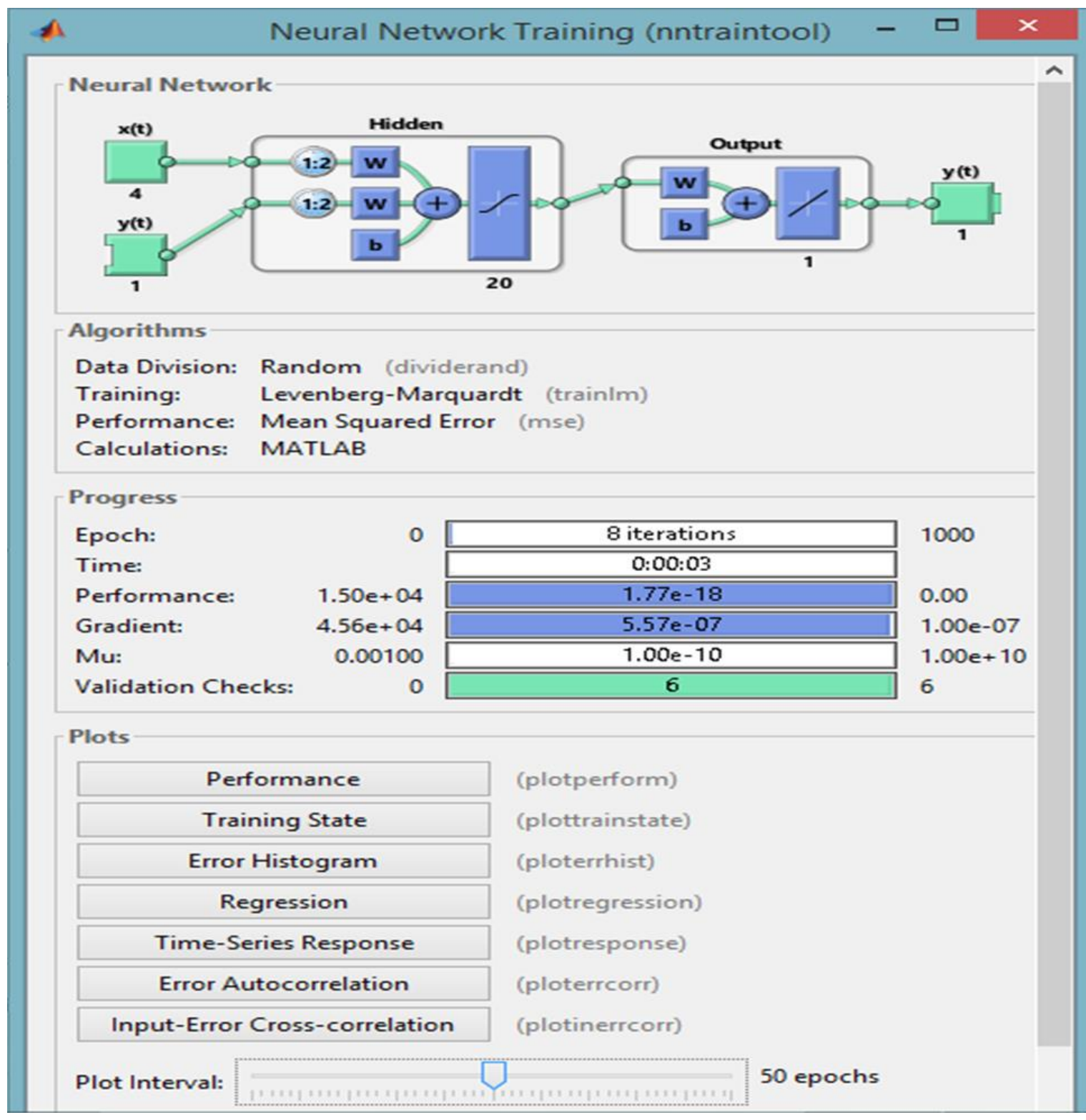

Figure 4: Matlab Training tool interface 


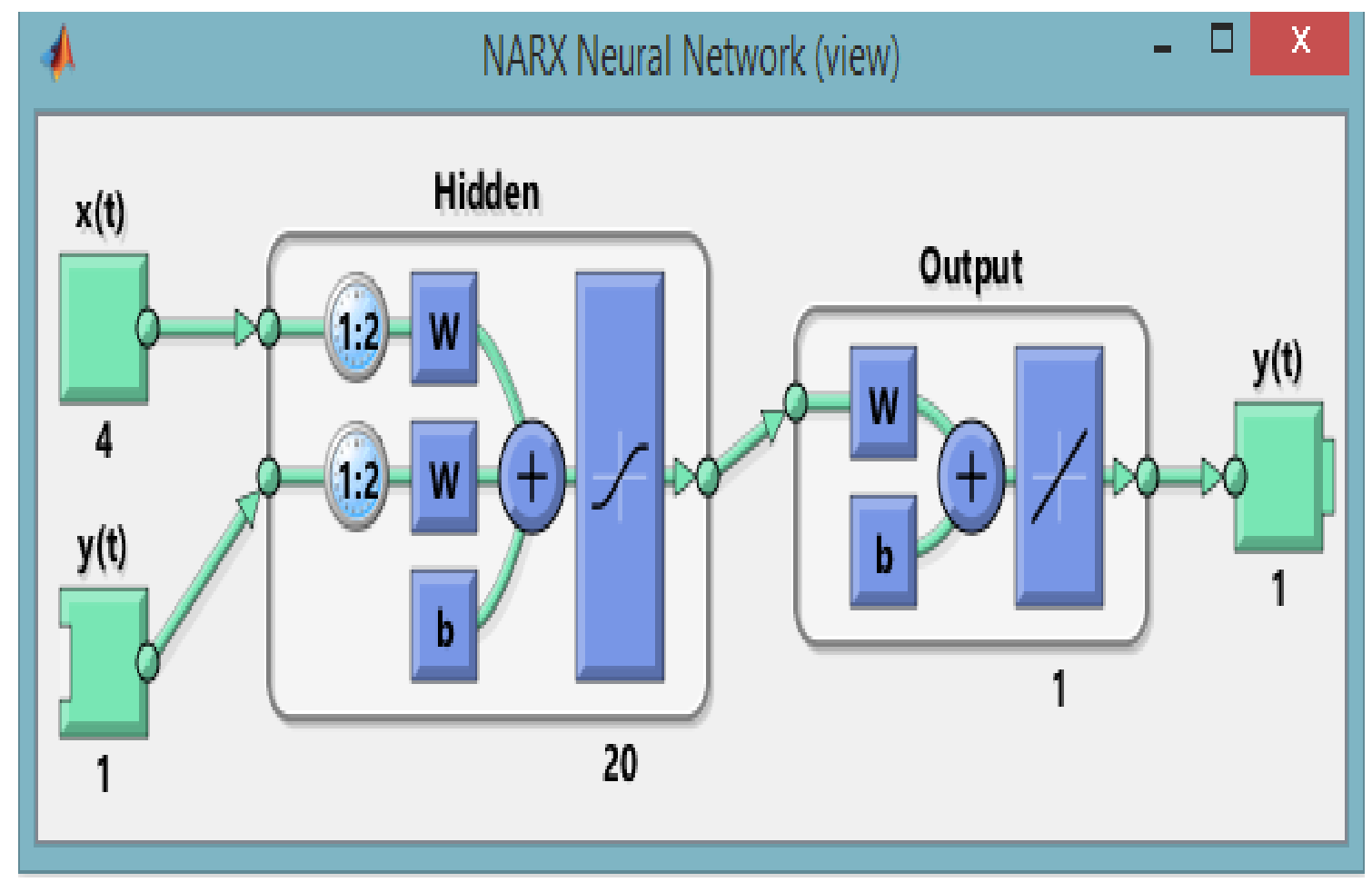

Figure 5: The designed NARX neural network for the Medium term Load prediction

The sigmoid transfer function was used in the hidden layer while the linear function was used in the output layer. The sigmoid function was applied because it shows well generalized learning characteristics, and the models with this function are always excellent and relatively more accurate. Training was carried out on the NARX neural network model until an optimum performance was attained with a reduced mean standard error (MSE). When an optimum performance was attained from the NARX network training, the code for the designed network was generated and saved to be used to run other sample data. The test data was also loaded to the network immediately after training and then the prediction results from these test data were obtained for year 2019.

\subsubsection{Performance Parameters}

The preciseness of every electricity load forecasting model is measured using the following parameters.

I. Mean Absolute Error (MAE): This type of error penalizes all errors equally, and is calculated using the function.

$$
M A E=\frac{1}{M} \sum_{i=1}^{M} \mid \operatorname{actual}(i)-\text { forecast }(i) \mid
$$

$M$ is the total number of examples or data points, actual( $i$ ) is the actual value of the ith term, forecast (i) is the forecasted value of the ith term.
II. Mean Absolute Percentage Error (MAPE): This type of error is the accepted standard for measuring the quality and accuracy of forecasted load. The mean absolute percentage error can be measured using the function;

MAPE $=\frac{1}{M} \sum_{i=1}^{M}\left|\frac{\operatorname{actual}(i)-\text { forecast }(i)}{\operatorname{actual}(i)}\right| \times 100 \%$

\subsubsection{Validation of the Network}

The performance plot of the proposed NARX NN model as obtained from Matlab software is as shown in Figure 6 . This plot was done automatically by the software using the training record of the network with the plotperf(tr) command. The training best epoch shows the iteration at which the validation performance reached a minimum. And from Figure 6 , the validation performance reached a minimum at epoch 2, with the training continuing for 6 more iterations before stopping the training process.

To check the correlation of the modelled output and the target, a regression plot was made. From the regression plot as shown in Figure 7 for the trained network, it can be seen that four plots were made for the training, validation, testing and for all of them together. It can be seen that the ' $R$ ' value for each of them is more than ' 0.9 ', with some being approximately ' 1 ' (one). The $\mathrm{R}$ values for the training, 
validation and the test data sets are ' 1 ', ' 0.88465 ' and 0.94665 respectively. This shows that there is almost exact relationship between the output obtained from the network and the targets and a good fit of the data points being used. This plot also helps to find out the outliers in the data points.

\subsection{Regression Model (MLR) for Load forecasting}

For the validation of the result obtained from the NARX ANN model, the forecast for electricity load demand for AMAC region of Abuja was also carried out using a Regression model, so that the result from this model can be compared to the ones obtained from the NARX ANN model. The regression model is a linear combination of parameters, and the independent variables. There are two types of linear regression, the simple linear regression and the multiple linear regressions.

In the simple linear regression of S-data points, there is only one independent variable $x_{i}$ and then two parameters $\sigma 0$ and $\sigma 1$ that give rise to a straight line known as the fitted regression line. The function for a simple regression is given by the function $[17$, eq. (6)]:

$$
y_{i}=\sigma_{0}+\sigma_{1} x_{i}+e_{i} i=1, \ldots, S
$$

Where $e_{i}$ is the error term representing variations in the dependent variables which were not accounted for, and are treated as random variables.
In multiple linear regression, more than one independent variables or function of variables are present. The regression model for a set of data having $m$ number of independent variables is as given [17] in eq. (14);

$$
y_{i}=\sigma_{0}+\sigma_{1} x_{1 i}+\cdots+\sigma_{m} x_{m i}^{2}+e_{i} i=1, \ldots N
$$

The accuracy and performance of regression models on the form of data generating process and its relation to the regression approach employed, with the best fit was obtained using the least squares method [17].

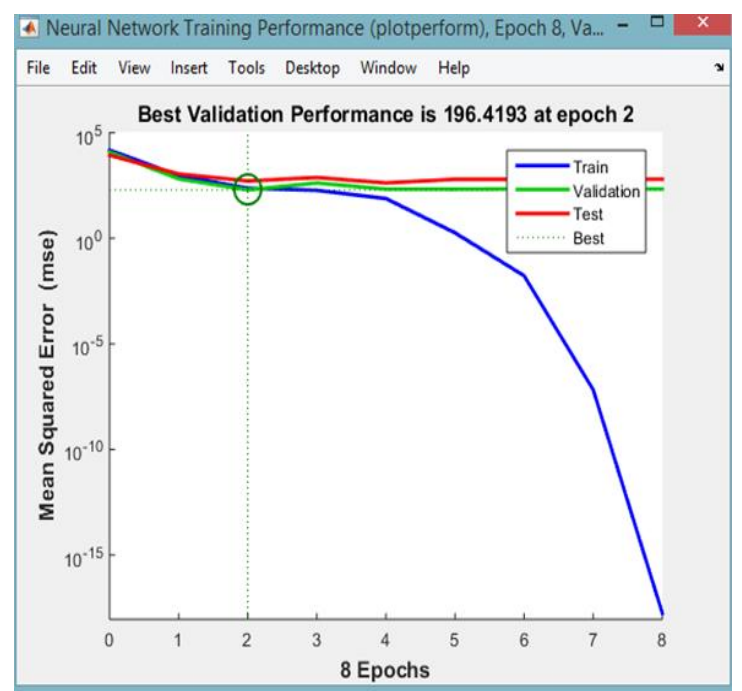

Figure 6: Performance plot of the Training of the proposed model

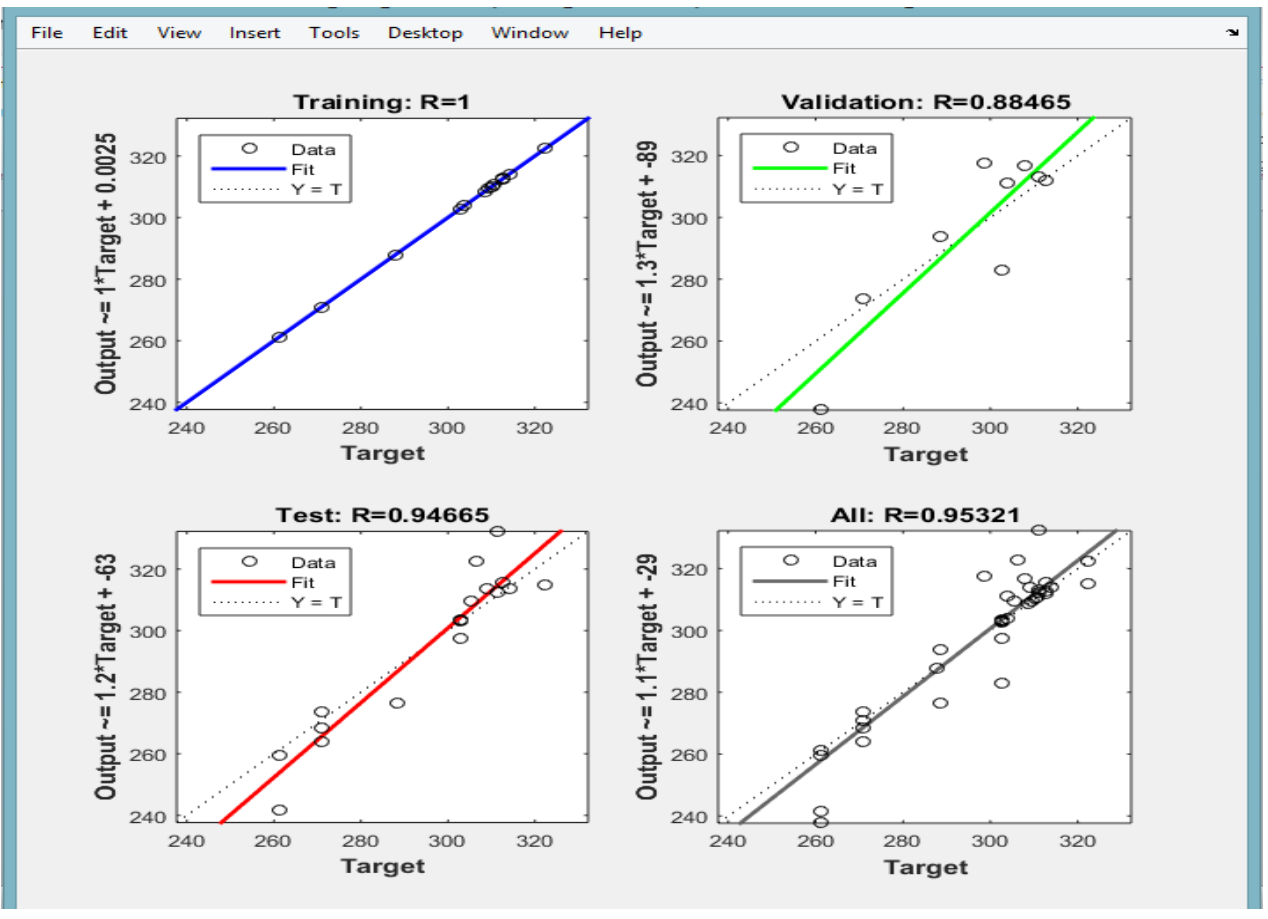

Figure 7: The regression plot of the trained network 
Since this work has more than one independent variable, the multiple linear regression model was applied in carrying out the load forecasting. In the model, the population and the average temperature condition of AMAC was used to obtain the energy demand. The previous load demand of AMAC from 2012 to 2017 was used to develop the regression model. The first model was used to forecast the future monthly temperature of AMAC for the next year, while the second was used to forecast the load demand of AMAC for that year forecasted.

\section{RESULTS}

Load demand in different areas of the world is dynamic in nature, having temporal seasonal and annual changes. The load data for this work was divided into months, and the prediction carried out with both the NARX-ANN and multiple linear regression (MLR) model. The predicted results obtained from the NARX ANN model and that obtained from multiple linear regression analysis are presented in tabular form in the sections below.

\subsection{Results obtained from the NARX ANN model}

The NARX neural network trained with a backpropagation network and with adaptive learning rate was able to predict the future monthly load of Abuja Area Municipal Council for one year ahead, given the accurate input of previous variables to the network. A sigmoid transfer function was employed in the hidden layer of the model, while in the output layer, the linear transfer function was used. The electricity load demand predictions were obtained from testing the NARX neural network on new 12 months for 3 years, the 2018 data and the predicted (2019 and 2020) temperature, humidity and population data of Abuja area municipal council (AMAC). The predicted Load demand of Abuja Municipal Area Council (AMAC) was plotted together with the Target Load against months represented with 1 to 12 for January to December, for year 2018 as shown in Table 1, with a mean squared error (MSE) of 116.7784 and an R value of 0.99669 , 0.92821 , and 0.91944 for the training, validation and test data respectively. The average mean absolute percentage error (MAPE) as obtained from the prediction using NARX neural network model was 0.00197 . With the mean absolute percentage error being very low, it means the network prediction is relatively accurate.
It can be seen that the peak load demand for Abuja Municipal Area Council (AMAC) in year 2018 occurred in May and October. During these months there is always a significant change in weather conditions from dry season to rainy season in May, and vice versa in October. With the weather changing from rainy season to dry (hot) season, residents of AMAC will need air conditioners to cool their houses and offices since residential houses and offices are the kind of houses mostly seen in AMAC. Also during the change of weather from dry season to rainy season when the weather will be cold, heaters and hot Jugs will also be needed for boiling water for bathing and drinking hot tea/coffee. These appliances require extra electrical energy thereby making it necessary to increase the electric power made available to the people of AMAC during these periods.

The performance of the NARX artificial Neural Network used for the prediction was plotted as shown in Figure 8. From Figure 8 it can be seen that the best performance was obtained at epoch 3 , after which there was 6 more iterations before the end of the prediction. The regression plots of the predicted values showing their correlation with the input data are as shown in Figure 9.

It can also be seen from Figure 9 that there is almost an exact correlation between the input data and the output data since the $\mathrm{R}$ values are close to or approximately ' 1 '.

Table 1: A table showing the predicted load and the MAPE of the ANN model

\begin{tabular}{cccc}
\hline MONTH & $\begin{array}{c}\text { Target } \\
\text { Load in } \\
\text { MW }\end{array}$ & $\begin{array}{c}\text { Forecasted } \\
\text { Load Using } \\
\text { ANN }\end{array}$ & $\begin{array}{c}\text { Mean Absolute } \\
\text { Percentage Error } \\
\text { (MAPE) }\end{array}$ \\
\hline 1 & 310.0285 & 310.3891 & 0.001162 \\
2 & 310.9235 & 311.2860 & 0.001165 \\
3 & 312.8532 & 313.2029 & 0.001117 \\
4 & 314.9658 & 315.1139 & 0.00047 \\
5 & 315.0754 & 315.8741 & 0.002529 \\
6 & 313.7345 & 314.6839 & 0.003017 \\
7 & 313.1025 & 313.6554 & 0.001763 \\
8 & 313.1253 & 313.6534 & 0.001684 \\
9 & 313.8213 & 314.6333 & 0.002581 \\
10 & 315.0215 & 316.3168 & 0.004095 \\
11 & 314.1025 & 314.2367 & 0.000427 \\
12 & 311.1256 & 312.2588 & 0.00197 \\
\hline & & & Average MAPE \\
& & & $=0.00197$ \\
\hline
\end{tabular}


Medium Term Electrical load Forecast of Abuja Municipal Area Council using ANN Method, E. C Ashigwuike, et al

\subsection{Load Forecasting Using Multiple Linear Regressions (MLR)}

Prediction using multiple linear regression was also done using Matlab software, the monthly average temperatures, monthly average humidity and monthly population of AMAC for year 2018 were used as inputs, and the monthly load demand was used as target. From the result obtained from multiple linear regression, the root mean square error value was 20.8302; the mean square error (MSE) obtained was 433.8981, while the $R$ value was 0.6258 . Table 2 shows the forecasted load values for the months in year 2018 as was obtained from the regression model. It can be seen that the predictions using multiple linear regression are farther away from the Target line meaning that the error value is high. Table 2 shows the forecasted load using multiple linear regression for AMAC with 2018 data and the calculated mean absolute percentage error (MAPE).

The average mean absolute percentage error obtained from load prediction using Multiple Linear Regression was 0.004545 as can be seen in Table 2 .

\subsection{Comparing the Two Models Used}

A Comparison of the two models based on the results obtained from the load forecast of Abuja Municipal Area Council using each of the models (NARX-ANN and the Multiple Linear Regression), showed that the NARX-ANN model used for prediction is relatively a better prediction model for medium term Load demand.
Table 2: The Predicted load and the MAPE using multiple linear regression.

\begin{tabular}{cccc}
\hline MONTH & $\begin{array}{c}\text { Target } \\
\text { Load in } \\
\text { MW }\end{array}$ & $\begin{array}{c}\text { Forecasted } \\
\text { Load Using } \\
\text { MLR }\end{array}$ & $\begin{array}{c}\text { Mean Absolute } \\
\text { Percentage Error }\end{array}$ \\
\hline 1 & 310.0285 & 311.8726 & 0.005913 \\
2 & 310.9235 & 311.8987 & 0.003127 \\
3 & 312.8532 & 314.3506 & 0.004763 \\
4 & 314.9658 & 316.0259 & 0.003354 \\
5 & 315.0754 & 316.8529 & 0.00561 \\
6 & 313.7345 & 314.9967 & 0.004007 \\
7 & 313.1025 & 314.0620 & 0.003055 \\
8 & 313.1253 & 314.0684 & 0.003003 \\
9 & 313.8213 & 315.5672 & 0.005533 \\
10 & 315.0215 & 317.4282 & 0.007582 \\
11 & 314.1025 & 314.9208 & 0.002598 \\
12 & 311.1256 & 313.0025 & 0.005996 \\
\hline & & & Average MAPE \\
& & & $=0.004545$ \\
\hline
\end{tabular}

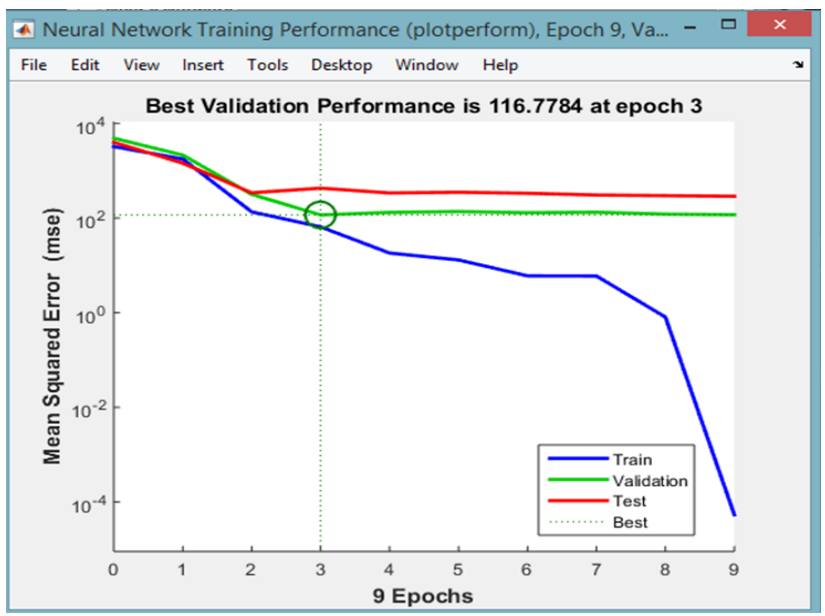

Figure 8: A performance plot of the NARX Neural Network prediction

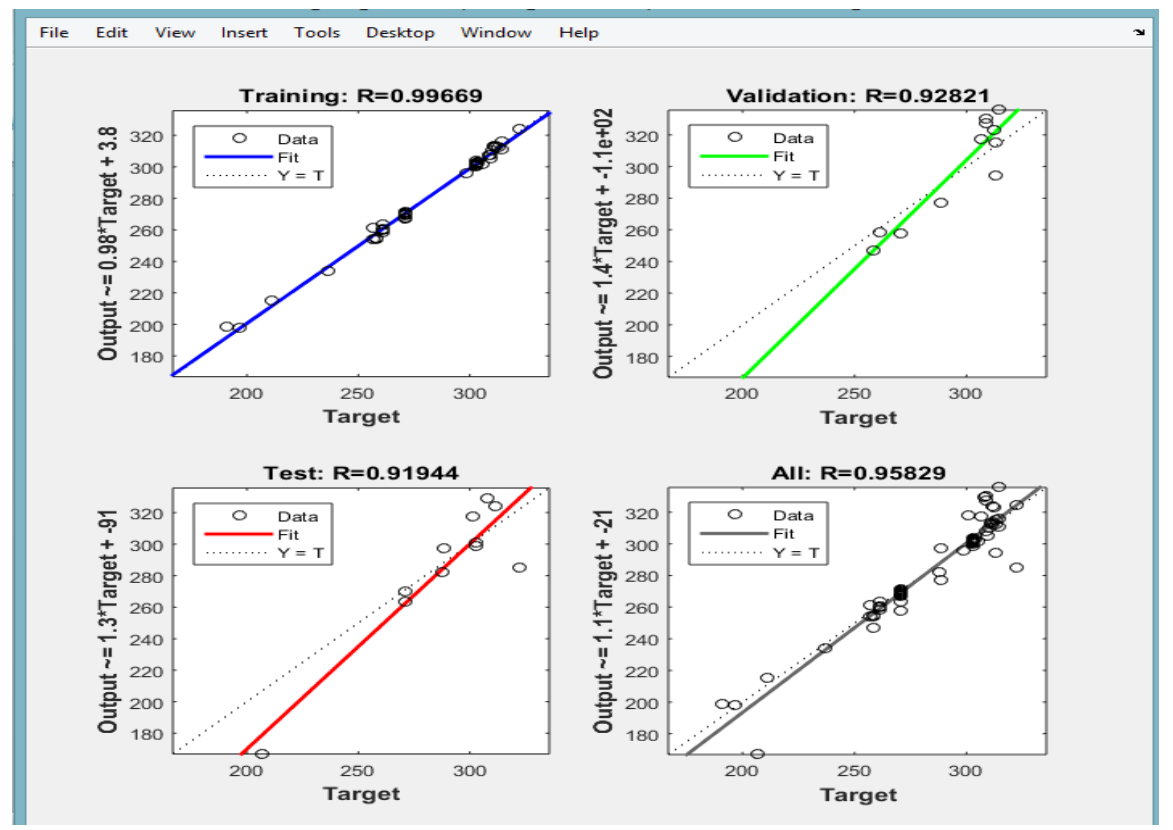

Figure 9: The regression plot of the load prediction 
A plot of the load demand predictions carried out by the NARX ANN model and multiple linear regression were plotted together with the Target load as shown in Figure 10. It can be seen from Figure 10 that the ANN prediction is closer to the target Load than the predictions from multiple linear regression, meaning that the ANN model is more accurate than the Multiple Linear Regression (MLR) model for medium-term electricity Load forecasting. Also from the mean absolute percentage error (MAPE), and the mean standard error (MSE) calculated from each of the models, it can also be seen that the MAPE and MSE from multiple linear regression is bigger than that obtained from the Artificial neural network model, showing that the prediction error is minimal in the NARX-ANN model as shown in Table 3.

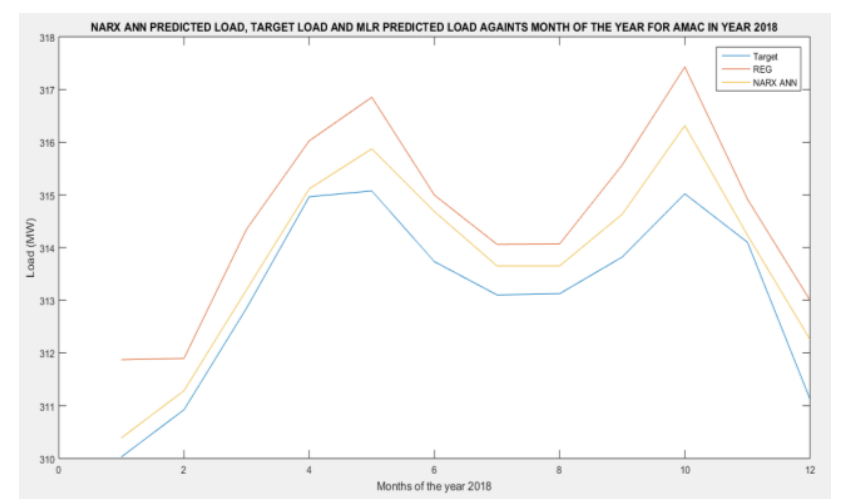

Figure 10: Predicted load of AMAC using ANN and using multiple linear regressions for year 2018

\begin{tabular}{ccc}
$\begin{array}{c}\text { Table 3: } A \text { summary of the results obtained from } \\
\text { both the }\end{array}$ MLR and the NARX-ANN models \\
\hline & $\begin{array}{c}\text { Multiple linear } \\
\text { regression }\end{array}$ & NARX-ANN \\
\hline R-Value & 0.6258 & 0.9194 \\
Ave. MAPE & 0.04545 & 0.00197 \\
MSE & 433.8981 & 116.7784 \\
\hline
\end{tabular}

It can also be seen that the R-value of the multiple regression model $(0.6258)$ is smaller than that of the NARX-ANN model (0.9194). Meaning that the multiple linear regression was not able to detect and determine all the relationships between the load demanded by AMAC and the factors affecting the load demand. Since the aim is to get a forecasted load as close as possible to the target load, the NARX ANN model from all indications and parameters considered showed a high degree of accuracy when compared to the multiple linear regression in medium-term load forecasting (MTLF). By standards accuracy level is when R-Value is at unity and from the result, the deviation from unity or error for ANN is only $8.06 \%$ whereas; that of MLR is $34.42 \%$.

These same processes are repeated using data for input variables and load variable to target a desired output for 2019, 2020 or even 2025. But care must be taken to project or estimate variables in line with percentage growth rate.

\section{CONCLUSION}

This work presented a model, capable of forecasting the Load demand of Abuja Area municipal council (AMAC) on a medium-term basis. This NARX-ANN based medium-term load forecasting technique which uses a three-layer feed forward neural network and a scaled back propagation algorithm for training is capable of predicting the Load demand of AMAC for the next 48 months. The historical data of the weather, population and the previous load demand of Abuja area municipal council (AMAC) were considered. To check the effectiveness and accuracy of the developed NARX-ANN model, multiple linear regression technique was also applied. The NARX-ANN model was able to perform better than the multiple linear regression model because it had to learn from training data the relationship between variables and their effect on the load demand thereby making a relatively precise forecast. The more the number of training cycles, the more the forecasting errors is improved. However over training the model leads to unfavourable results. It can also be seen from the NARX-ANN model that if the number of input variables are increased, the accuracy of the forecast is also increased. It can be concluded therefore that the artificial neural network model is better for mediumterm load forecasting, and generally, it can also be concluded that artificial intelligence methods are better than the conventional methods for electric load demand forecasting.

\section{REFERENCES}

[1] Saeed, B. and Ossama A., "Neural Network Integrated with Regression Methods to forecast Electrical load", International Conference on Electrical, Electronics and Biomedical Engineering. Penang, Malaysia, ICEEBE. 2012: pp. 60-65.

[2] Ihedioha, A. C. and Eneh I. I., "Load forecasting using artificial neural networks " International Journal of Research in Advanced Engineering and Technology Vol. 2, Number 3, 2016, pp. 75-78. 
[3] Irisarri, G. D., Widergren S. E. and Yehsakul P. D., "On-line load forecasting for energy control center application " IEEE Transactions on Power Apparatus Systems Vol. 101, 1982, pp. 71-78.

[4] Hill, T., M. O'Connor and W. Remus (1996). "Neural networks models for time series forecasts." Manage Science, Vol 5, 1996, pp.1082-1092.

[5] Amjady, N, "Short-term hourly load forecasting using time series modeling with peak load estimation capability." IEEE transaction on power systems, Vol 16, 2001, pp. 798-805.

[6] Almeshaiei, E. and Soltan H., "A methodology for Electric Power Load Forecasting." Alexandria Engineering Journa/ Vol. 50, 2011, pp. 137-144.

[7] Filik, U.B.O., Gerek, N., and Kurban M., "A novel modeling approach for hourly forecasting of longterm electric energy demand." Energy Conversion and Management Vol. 52, 2011, pp. 199-211.

[8] Feinberg E.A., Genethliou D. Load Forecasting. In: Chow J.H., Wu F.F., Momoh J. (eds) Applied Mathematics for Restructured Electric Power Systems. Power Electronics and Power Systems. Springer, Boston, MA, 2005.

[9] Singh, A. K. N., Ibraheem, S. K., Muazzam M., and Chaturvedi D. K., Load Forecasting Techniques and Methodologies: A review. 2012 2nd International Conference on Power, Control and Embedded Systems. New Delhi: 2012, pp. 631640.

[10] Arjun B., Kumar G., Sudhansu K. M., "A Review of Short Term Load Forecasting using Artificial Neural Network Models", International Conference on Intelligent Computing, Communication \& Convergence (ICCC-2014),
Bhubaneswar, Odisha, India, Procedia Computer Science Vol 48, 2015 pp. 121 - 125.

[11] Amera I., Melhum L. O., Sozan A. M., " Short Term Load Forecasting using Artificial Neural Network", International Journal of Soft Computing and Engineering, Volume-3 Issue-1, March 2013.

[12] Park D.C., M.A., El-Sharkawi R. J. Marks L. E. A., and Damborg M.J., "Electric Load Forecasting Using An Artificial Neural Network", IEEE Transactions on Power Systems, Vo1.6, Number 2, May 1991 pp. 442 - 449.

[13] Kuhba, H. and Hassan A. H. (2016). "Power system short-term load forecasting using artificial neural networks." International Journal of Engineering Development and Research Vol 4, Number 2, 2016, pp. 78-88.

[14] Zina, B., C., Ahmed R. and Haritza C., "A Nonlinear Autoregressive Exogenous (NARX) Neural Network Model for the Prediction of the Daily Direct Solar Radiation." Energies Vol 620, 2018.

[15] World Population Review: Available at https://worldpopulationreview.com/worldcities/abuja-population/ accessed 27/05/2019 3:39 pm.

[16] Dorffner, G., "Neural networks for time series processing." Neural Network World Vol 6, Number 4, 1996, pp. 447-468.

[17] Kumaran, J. K. and Ravi G., "A Simple Regression Model for Electrical Energy Forecasting." International Journal of Advanced Research in Electrical, Electronics and Instrumentation Engineering Vol. 5, Number 2, 2010, pp. 20-30. 